\section{Comment on "Auditory frequency sensitivity of human newborns: Some data with improved acoustic and behavioral controls"}

\author{
WILLIAM A. YOST \\ Parmly Hearing Institute, Loyola University \\ Chicago, Illinois 60626
}

Catherine Weir's article, "Auditory frequency sensitivity of human newborns: Some data with improved acoustic and behavioral controls," offers a procedure which should help improve greatly one's ability to use results from the auditory response cradle or "cribogram" in a meaningful manner. It is because of this strength, and my hope that the procedures will be used, that I would like to comment on a weakness in the discussion of Experiment 2.

The discussion of Experiment 2 centers on the "thresholds" obtained across frequencies which were all about the same value. Weir offers a variety of hypotheses pertaining to the outer and middle ears to explain these results. The hypotheses are offered to explain the discrepancy between adult thresholds which vary with frequency and the thresholds from the infants which do not. Her levels of between 33 and $42 \mathrm{~dB}$ SPL above ambient noise level appear to be of an intensity which gives some credance to the assumption that the infant thresholds are analogous to those obtained when adults are asked to detect tones. The problem, to me, lies primarily in her computation of the levels. The ambient noise level is stated as being broadband with a level of $45 \mathrm{~dB}$ SPL while the thresholds of the tones ranged from 78 to $87 \mathrm{~dB}$ SPL. It is not appropriate to subtract the broadband noise level from the tonal levels in order to estimate the level of the tones above the ambient noise level in the ear canal. This type of signal-tonoise ratio should be based on some measure of the noise in the spectral region of the tone which reflects the masking effect of the noise on the tone. Measures such as spectrum level and level in a critical band are the two most frequently used measures. This problem is even more serious when one considers the author's statement that "virtually all of the energy in the external auditory canal was less than $20 \mathrm{~Hz}$." That is, there was little, if any, ambient noise in the spectral region of the tones $(125-4,000 \mathrm{~Hz})$. Therefore, the level of the tones at threshold ranged from approximately 78 to $87 \mathrm{~dB}$ above the level of any noise which could have caused masking. That is, the thresholds were 78 to $87 \mathrm{~dB}$ SPL, not 33-42 dB SPL.

I wonder if, at these high levels, the responses measured in the crib are at all like "detection responses" which one hopes to measure in an adult. Could not the responses be more like startle, annoyance, irritation, etc., to a novel or even mildly noxious stimulus? The psychophysical results on suprathreshold signals (loudness, annoyance, masking, discomfort level, etc.) all show that the signal level required for these types of responses is approximately the same (within $15 \mathrm{~dB}$ ) across the frequency range of $125-4,000 \mathrm{~Hz}$. Thus, I maintain that another hypothesis which should be considered to explain the results of Experiment 2 in Weir's paper is that the infants are responding to superthreshold signals in a manner similar to the way in which adults respond to superthreshold signals. Furthermore, the infant's response might be one of startle or reaction to a novel or noxious stimulus in the nursery environment.

My purposes, therefore, in writing this comment were threefold: (1) to commend Dr. Weir on bringing some control to the cribogram procedure, (2) to indicate that the levels required to elicit responses in the procedure are more accurately described as $78-87 \mathrm{~dB}$ SPL rather than 33-42 dB, and (3) to suggest that the results might reflect the infant's response to a suprathreshold acoustic event. I believe these comments are important to consider if the article will have the impact that I hope it will have on the testing of hearing in the newborn.

\section{REFERENCE}

WeIR, C. Auditory frequency sensitivity of human newborns: Some data with improved acoustic and behavioral controls. Perception \& Psychophysics, 1979, 26, 287-295.

(Received for publication December 7, 1979 accepted December 11, 1979.) 